\title{
Original Article (short paper) \\ Intensity-dependent effects of cycling exercise on corticospinal excitability in healthy humans: a pilot study
}

\author{
Isis Suruagy \\ Adriana Baltar \\ Universidade Federal de Pernambuco, Recife, PE, Brasil \\ Luis Paulo Gomes \\ Universidade Tiradentes, Aracajú, SE, Brasil \\ Marina Berenguer \\ Armele Dornelas \\ Kátia Monte-Silva \\ Universidade Federal de Pernambuco, Recife, PE, Brasil
}

\begin{abstract}
Aims: the aim of this study was to verify the effects of different intensities of locomotor exercise on corticospinal excitability. Methods: 18 healthy subjects ( $27.6 \pm 6.5$ years, ) participated in a design study of three different exercise protocols on a cycle ergometer: (i) $10 \mathrm{~min}$ at $75 \% \mathrm{Wmax}$ (high intensity); (ii) $15 \mathrm{~min}$ at $60 \% \mathrm{Wmax}$ (moderate intensity) or (iii) $30 \mathrm{~min}$ at $45 \% \mathrm{Wmax}$ (low intensity). The protocols of lower body cycling were assigned in random order in separate sessions. A control session was done with subjects at rest. Corticospinal excitability was assessed before (baseline) and every $5 \mathrm{~min}$ for $15 \mathrm{~min}$ after the end of exercise/rest (time: $0,5,10$ and 15) by measurement of the motor evoked potential (MEP) elicited by transcranial magnetic stimulation in the relaxed first-dorsal interosseus muscle. Results: Compared to the resting session, a significant decrease $(64 \%)$ in the motor evoked potential amplitudes was found only in the session of exercise of high intensity. This result seems depend on the level of physical activity of subject. No change was found after rest, low and moderate exercises. Conclusions: These findings suggest that changes in the corticospinal excitability depend on exercise intensity and level of physical activity of subjects.
\end{abstract}

Keywords: transcranial magnetic stimulation, motor córtex, motor evoked potentials, exercise

\section{Introduction}

The specific mechanisms that influence the central nervous system (CNS) changes during or after different types of exercises are controversial ${ }^{1,2}$. Distinct modes of exercise may result in differential afferent feedback signals to CNS and/or direct central effects via intrinsic brain modulations and conscious perceived of effort ${ }^{3,4}$. However, great variations among exercise modalities in the amount of muscle work, and the consequent cardiorespiratory demands and other factors such as cerebral oxygenation consumption rate may also explain the differential corticospinal responses after dynamic vs. static exercise ${ }^{5,7}$.

There have been some reports in which transcranial magnetic stimulation (TMS) has been used to test the effects of physical exercise on corticospinal excitability ${ }^{8,9}$. Its effects on the responsiveness of corticospinal cells appear to be dependent on the type of exercise (dynamic vs static) ${ }^{5,8}$. Some of dynamic exercises (exercises involving multiple limb muscles) do not change the amplitude of motor evoked potentials (MEPs) $)^{5,10}$. However, reduction in the amplitude of MEPs for at least $20 \mathrm{~min}$ after fatiguing exercise has also been documented ${ }^{11,12}$. Decrease in the amplitudes of MEPs after fatiguing exercise, a phenomenon called post-exercise MEP depression, and an increase in MEP amplitudes after non-fatiguing exercise, a phenomenon called post-exercise MEP facilitation are shown in static exercise, but are not observed in some dynamic exercise $^{10,12}$. Other studies suggested some differences in cortical and spinal responses to dynamic exercise (e.g. locomotor) versus static exercise (e.g. single-joint $)^{3,5,8,13}$.

There is little evidence about whether the effect of exercise on corticospinal excitability is also dependent on the exercise intensity. To the best of our knowledge, Höllge, Kunkel, Ziemann, Tergau, Geese, Reimers ${ }^{14}$ were the first who evaluated the influence of different dynamic exercise with different intensities on the TMS-induced MEP amplitude. They demonstrated distinct responses on excitability mainly between exhaustive (high intensity) and non-exhaustive exercise (low intensity). Furthermore, few researches have investigated corticospinal excitability after dynamic non-exhaustive exercise with different intensities. Therefore, the present study was designed to test the hypothesis that differences in MEPs amplitude would occur after dynamic non-exhaustive cycling exercise with different intensities.

\section{Experimental Procedures}

\section{Subjects}

Eighteen healthy subjects participated in this crossover study. The level of physical activity of subjects were categorised into very active ( 2 women and 3 men), active ( 8 women) and irregularly 
active (4 women and 1 men) by International Physical Activity Questionnaire (IPAQ) ${ }^{15,16}$. All participants were informed of the procedures and risks before giving written informed consent to participate in the research. Approval for study procedures was obtained from the Institutional Research Ethics Committee and was performed in accordance with the Declaration of Helsinki.

\section{Maximal incremental exercise test}

Before the experimental sessions (session 1), the subjects were submitted to maximal incremental exercise test to find out their maximal tolerated power (Wmax). For the test, participants sat on the cycle ergometer (ERGO-FIT® model 167 cycle, Pirmansens, Germany) with similar riding position (saddle and handlebar height and position) and were asked to begin cycling at $70 \mathrm{rpm}$ during a warm-up phase ( $3 \mathrm{~min})$ at a work load of $15 \mathrm{~W}$. The work rate was then increased by $25 \mathrm{~W}$ per minute until exhaustion when participants could not keep a cadence of $70 \mathrm{rpm}$ for 5 seconds or when the subjects voluntarily terminated the test. During the test, the subjects were verbally encouraged to continue for as long as possible. Wmax was defined as the highest work load sustained by the subject for longer than $1 \mathrm{~min}$.

\section{Experimental design}

The protocol included three experimental sessions (sessions 2, 3 and 4) which were conducted in a pseudo-randomized (counterbalanced sequence) crossover design. In each session, one of three different exercise intensities on a cycle ergometer was tested: (i) $10 \mathrm{~min}$ at $75 \% \mathrm{Wmax}$ (high intensity); (ii) $15 \mathrm{~min}$ at $60 \% \mathrm{Wmax}$ (moderate intensity) or (iii) $30 \mathrm{~min}$ at $45 \% \mathrm{Wmax}$ (low intensity). A control session (session 5) was done with subjects at rest, the subjects were asked to stay sit without move the hands. The sessions were separated by at least two days.

In all experimental sessions, during the exercise, the heart rate (HR) was continuously registered using an HR recorder (Polar RS800, Polar Electro, Kempele, Finland). In addition, the Borg Rating of Perceived Exertion (RPE) ${ }^{17}$ was used to estimate wholebody perceived exertion. Every three minutes during exercise, the RPE was displayed in front of the subjects who were asked to point the number that the best described their perceived exertion (number 7 represents unloaded cycling while number 19 indicates an exertion similar to exhaustive cycling) ${ }^{17}$.

The volunteers were instructed to refrain from vigorous activities and to avoid the consumption of caffeine, alcohol and tobacco for $24 \mathrm{~h}$ prior to each session. For all exercise time, the laboratory temperature $\left(19 \pm 1^{\circ} \mathrm{C}\right)$ remained constant in order to minimize environmental influence.

\section{Monitoring of motor corticospinal excitability}

Before and after each session, the corticospinal excitability was assessed by MEPs elicited by TMS. For this, the subjects were seated in a comfortable chair with head and arm rests. Single-pulse
TMS was applied using a figure-of-eight magnetic coil ( $7 \mathrm{~cm}$ diameter) connected to a magnetic stimulator (Neurosoft Ltd., Russia; peak magnetic field $=2.2$ tesla). The coil was held tangentially to the skull, with the handle pointing backwards and laterally at an angle of $45^{\circ}$ from midline in the right hemisphere. The optimal position was defined as the site where stimulation resulted in the largest MEPs. Surface EMG was recorded from the left first dorsal interosseous (FDI) with $\mathrm{AgCl}$ electrodes in a belly-tendon montage. The signals were amplified and filtered with a time constant of $80 \mathrm{~ms}$ and a low-pass filter of $5.0 \mathrm{~Hz}$, then digitized at an analogue-to-digital rate of $20 \mathrm{kHz}$ and further relayed into a laboratory computer using the Neuro-MEP-Micro software (Neurosoft Company, Russia). The intensity was adjusted to elicit, on average, baseline MEPs of $1 \mathrm{mV}$ peak-to-peak amplitude and was kept constant for the post-stimulation assessments. During the registers, EMG was recorded online in order to ensure the rest of FDI. Twenty MEPs were recorded at frequency of 0.25 $\mathrm{Hz}$ at baseline and every 5, 10 and 15 minutes.

The design of the experiment is shown in figure 1.

\section{Statistical analysis}

Individual MEP amplitude means were calculated for each time bin, including baseline and post-exercise/rest time points. The post - exercise/rest MEPs were normalized intra-individually and are given as baseline ratios. In order to verify any difference in the conditions before each session one - way ANOVA were employed.

A repeated measure ANOVA was calculated using the withinsubject factors "time course" (baseline, 0,5,10 and 15min) and "exercise protocols" (low, moderate, high intensity and rest) and the dependent variable MEP amplitude. Also by repeated measure ANOVA, the behaviour of excitability after physical exercise was also analysed considering the level of physical activity of the subjects. Post hoc analyses were performed with a student paired-samples $t$ test when appropriate. The Mauchly test of sphericity was checked and the Greenhouse-Geisser correction was performed, if necessary. The data were analyzed using the program SPSS (version 18.0). A p value of $<0.05$ was considered significant for all statistical analysis.

\section{Results}

Table 1 shows the characteristics of sample and averaged values for HR and RPE obtained during each exercise protocols and work load correspondent are given in the table 2 .

The results of the one-way ANOVA showed that MEP amplitudes did not differ significantly in baseline measurements among the sessions. The repeated measures ANOVA revealed a significant effect of time $(\mathrm{F}=5.968, \mathrm{p}=0.002)$ and exercise protocol $(\mathrm{F}=3.473, \mathrm{p}=0.039)$, but not for interaction exercise protocol $(\mathrm{F}=1.929, \mathrm{p}=0.102)$. As showed by the post hoc test, exercise of high intensity decreases significantly corticospinal excitability until $15 \mathrm{~min}$ after the end of exercise when compared to baseline and resting condition. Compared to baseline MEPs, a significant depression in corticospinal excitability was observed also after exercise of low-intensity. No change on excitability was found after rest or moderate-intensity exercise (Figure 2). 

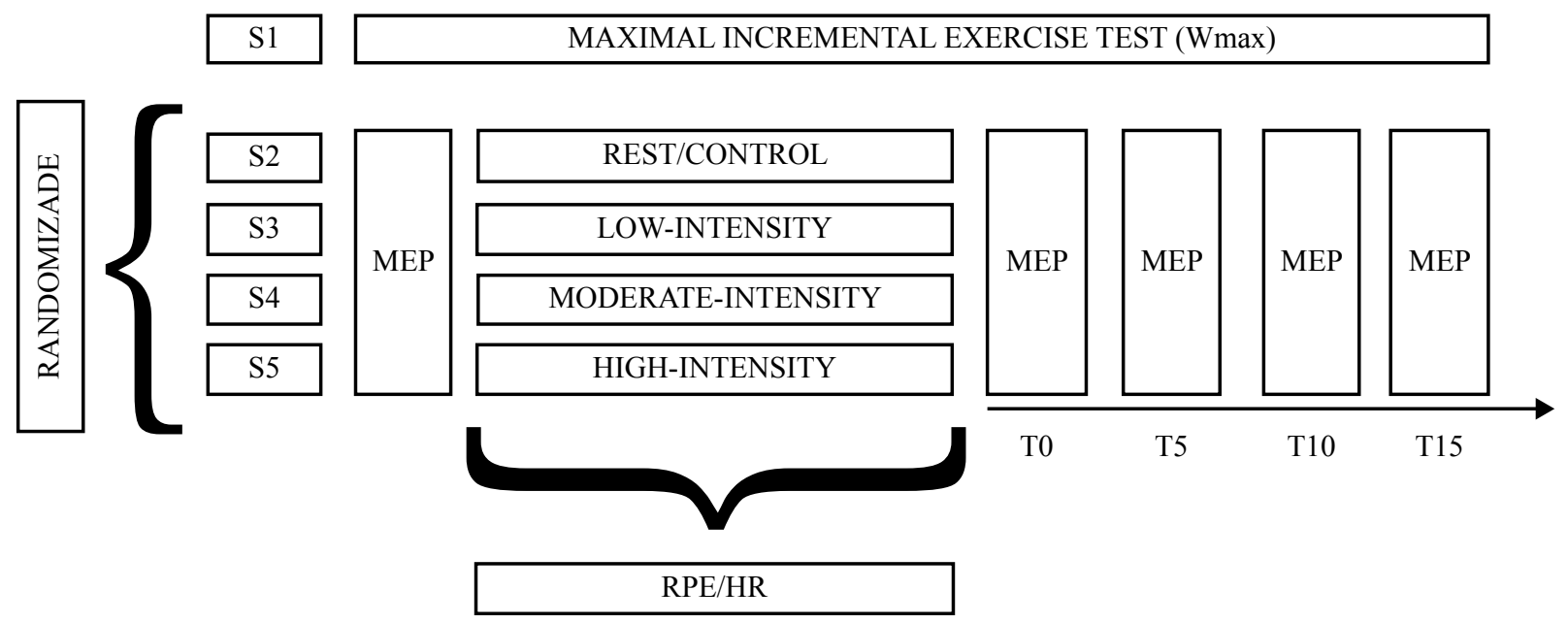

Figure 1.Experimental course. In session 1, subjects were submitted to maximal incremental exercise test to find out their maximal tolerated power (Wmax). In sessions 2,3 and 4, the effect of different exercise protocols on motor cortex excitability, as monitored via motor evoked potential (MEP - 20 stimulus) elicited by single pulse transcranial magnetic stimulation (TMS), was explored. Specifically, high (10min at $75 \%$ Wmax); moderate ( $15 \mathrm{~min}$ at $60 \% \mathrm{Wmax}$ ) and low intensity (30 min at 45\% Wmax) were compared with control session (session 5) with subjects at rest. The excitability was monitored every $5 \mathrm{~min}$ for one quarter of an hour (T0, T5, T10 and T15) after the end of exercise/rest. RPE - rating perception effort, HR - heart rate, MEP - motor evoked potential.

Table 1.Participants characteristics

\begin{tabular}{|c|c|}
\hline Variable & \\
\hline Age, in years mean $( \pm S D)$ & $27.6( \pm 6.5)$ \\
\hline $\begin{array}{l}\text { Gender } n(\%) \\
\text { Male } \\
\text { Female }\end{array}$ & $\begin{array}{l}4(22.2 \%) \\
14(77.7 \%)\end{array}$ \\
\hline $\mathbf{B M I}$, in $\mathbf{K g} / \mathbf{c m}^{2}$ mean $( \pm \mathrm{SD})$ & $21.5( \pm 1.82)$ \\
\hline $\begin{array}{l}\text { Physical activity }{ }^{1} \mathrm{n}(\%) \\
\text { Irregularly active } \\
\text { Active } \\
\text { Very active }\end{array}$ & $\begin{array}{l}5(28 \%) \\
8(44 \%) \\
5(28 \%)\end{array}$ \\
\hline $\begin{array}{l}\text { Wmax }^{2} \text { mean }( \pm \mathrm{SD}) \\
\text { Irregularly active } \\
\text { Active } \\
\text { Very active }\end{array}$ & $\begin{array}{l}197.7( \pm 60.2) \\
153( \pm 48.3) \\
178( \pm 14.5) \\
265( \pm 62.7)\end{array}$ \\
\hline
\end{tabular}

${ }^{1}$ Determined by International physical actitvity questionnaire - IPAQ; ${ }^{2} \mathrm{De}-$ termined by the maximal incremental exercise test. $\mathrm{W}-$ Power output. BMI - body mass index

Table 2. Mean ( \pm standard deviations) of heart rate (HR) 1, Borg rating of perceived exertion (RPE) 2 and power (watts) during exercise protocols on a cycle ergometer.

\begin{tabular}{|c|c|c|c|}
\hline Exercise Protocols & HR $^{1}$ & $\mathbf{R P E}^{2}$ & Power \\
\hline High intensity (10min at $75 \% \mathrm{Wmax})$ & $\begin{array}{c}163.3 \\
( \pm 10.6)\end{array}$ & $\begin{array}{c}15.5 \\
( \pm 2.0)\end{array}$ & $\begin{array}{c}148.3 \\
( \pm 45.1)\end{array}$ \\
\hline $\begin{array}{l}\text { Moderate intensity }(15 \mathrm{~min} \text { at } 60 \% \\
\text { Wmax) }\end{array}$ & $\begin{array}{l}149.4 \\
( \pm 9.2)\end{array}$ & $\begin{array}{c}13.0 \\
( \pm 1.4)\end{array}$ & $\begin{array}{c}118.6 \\
( \pm 36.1)\end{array}$ \\
\hline Low intensity ( $30 \mathrm{~min}$ at $45 \% \mathrm{Wmax}$ ) & $\begin{array}{l}135.0 \\
( \pm 2.9)\end{array}$ & $\begin{array}{c}10.2 \\
( \pm 1.0)\end{array}$ & $\begin{array}{c}89.0 \\
( \pm 27.1)\end{array}$ \\
\hline
\end{tabular}

${ }^{1} \mathrm{Bpm}$, beat per minute; ${ }^{2}$ score of Borg scale

RPE - rating perception effort e HR - heart rate

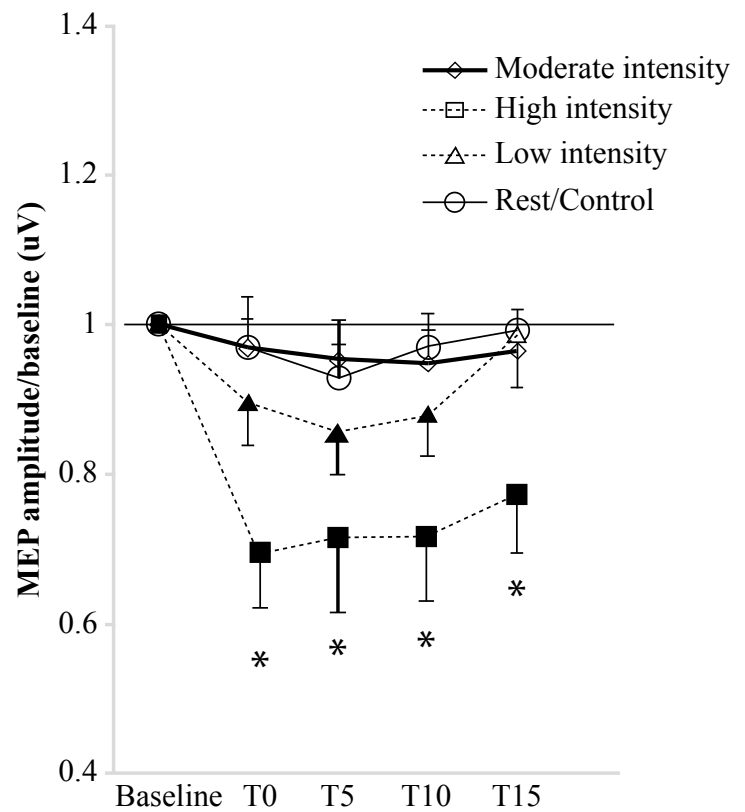

Figure 2. Intensity-dependent effects of physical exercise on corticospinal excitability. Shown are the mean $( \pm \mathrm{SE})$ of baseline-standardized motor evoked potential (MEP) amplitudes before (baseline) and after $(0,5,10$ e $15 \mathrm{~min})$ exercise/rest condition. Filled symbols indicate significant deviations of the post-exercise/rest MEP amplitudes from baseline values; *significant deviations of rest condition.

Figure 3 plots the mean $( \pm$ SE) of baseline-standardized MEP amplitude change over the course of the session of high-intensity (3A), moderate-intensity (3B) and low-intensity exercise (3C) for groups of subjects classified as very active, active or irregularly active, according to IPAQ. The post hoc test revealed a significant corticospinal excitability reduction only after session with exercise of high - intensity in very active subjects when compared to baseline condition and to the resting/control session. 

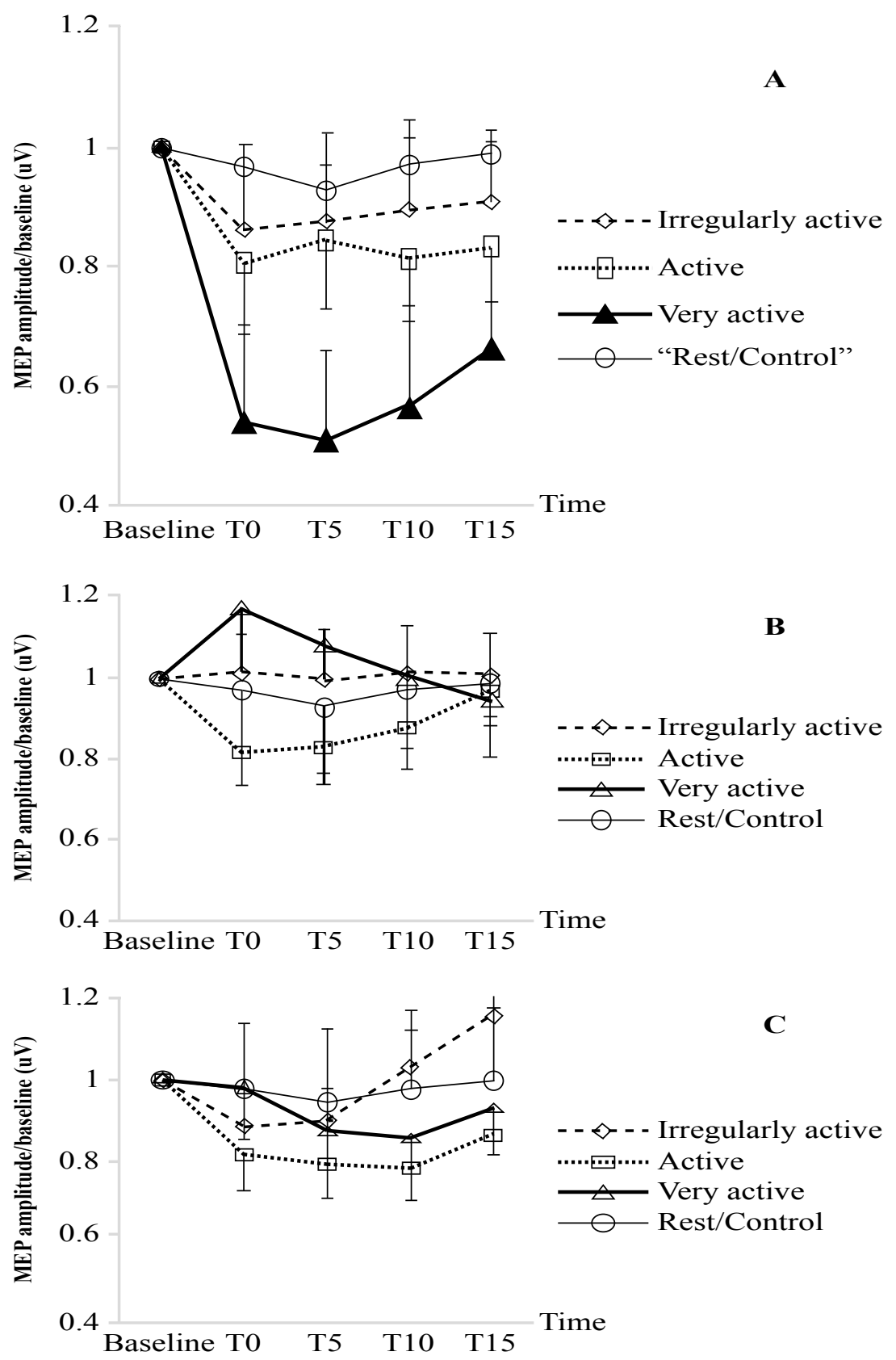

Figure 3. Intensity-dependent effects of physical exercise on corticospinal excitability are depend on the physical activity level of subjects. The time course plots show the effect of different intensities of exercise, high-intensity (3A), moderate-intensity (3B) and low-intensity (3C). Shown are the mean $( \pm$ SE) of baseline-standardized motor evoked potential (MEP) amplitudes before (baseline) and after $(0,5,10$ e 15 min) exercise/rest condition. Filled symbols indicate significant deviations of the post-exercise/rest MEP amplitudes from baseline values; *significant deviations of rest condition

\section{Discussion}

The present study evaluated the effect of a single session of dynamic exercise with three different intensities modifies in the corticospinal excitability. The results demonstrate a significant decrease in the MEP amplitude after high intensity exercise for $10 \mathrm{~min}$. No changed was found after low and moderate-intensity exercise.

This pattern of change after high intensity exercise is similar to that observed after sustained single-joint contraction $^{18,19}$ fatiguing running exercise ${ }^{20}$, strength exercises ${ }^{14}$ and after maximal incremental treadmill exercise ${ }^{21}$. Most studies that found a reduction of the MEP amplitude are those which used fatiguing exercise ${ }^{12,14,20}$. Despite the intense exercise $(75 \%$ Wmax) used in our study not be considered fatiguing, the intensity and duration that were used probably were enough to promote accumulation of fatigue metabolites and corresponding increased firing of muscle metabosensitive receptors, or other acute exercise-induced responses that are common to fatiguing exercise $^{5}$. Although the precise cellular mechanisms underlying post-exercise MEP depression are unclear, Sammi, Wassermann, Hallet $\mathrm{t}^{22}$ hypothesize that exercise may modify synaptic transmission within the motor cortex for several minutes in a way 
similar to that in which high-frequency microstimulation of a synaptic pathway leads to depressed transmission.

In contrast to our results, previous TMS studies ${ }^{23-24}$ that used cycling exercise of similar high intensity ( $80 \% \mathrm{Wmax}$ ) and longer duration ( $\sim 45 \mathrm{~min}$ ) reported no changes in MEP responses measured either in vastus lateralis ${ }^{23}$ or rectus femoris muscles ${ }^{24}$. One reason for this discrepancy might be due the place where the corticospinal excitability was evaluated, in the cortical representation area of non-exercised muscle (our study) in contrast to exercised muscle (previous studies) ${ }^{23-24}$. However, contrary to this hypothesis, Takahashi et al. ${ }^{25}$ showed that intense exercise of leg muscles leads to pronounced effects on the corticospinal and corticocortical excitability of two non-exercised arm muscles (FID and biceps brachi). Therefore, exhaustive exercise of large muscle groups might cause a widespread cerebral deoxygenation and affects the excitability of circuits in the non-exercised area of motor cortex ${ }^{26}$.

In contrast to the effects of high-intensity exercise, no change on corticospinal excitability was found after low - and moderate-intensity exercise in our study. Similar findings have been demonstrated by previous studies ${ }^{27-28}$. It is likely that nonfatiguing dynamic exercises of low and moderate intensity are unable to influence non-exercised muscles cortical area. However, a recent study reported that even when measured in the muscles directly involved in the exercise, a period of sustained cycling did not significantly influence motor cortex excitability ${ }^{10}$. This response pattern in corticospinal responsiveness differs from that observed after non-fatiguing single-joint contraction, which corticospinal excitability increases markedly ${ }^{22,29}$. For example, Samii, Wassermann, Ikoma, Mercuri, Hallett ${ }^{29}$ observed the amplitude of MEPs induced by TMS from the resting muscle after 30-second periods of non-fatiguing isometric exercises of the extensor carpi radialis was on average more than twice the pre-exercise value. Given that the amount of muscle work and associated oxygen demands are considerable different to the two modes of exercise (single-joint vs. locomotor exercises), these and other differences in their associated systemic physiological consequences may explain the contrasting corticospinal responses ${ }^{10}$.

The differences among the systemic physiological consequences, such as hyperthermia, arterial hypoxemia, decreases in cerebral oxygenation, respiratory muscle loading, and brain catecholamines, occasioned by different exercise intensities may also help to explain the variable responses of central nervous system drive to high and low/moderate levels of exercise intensity in our study. This seems to happen because different modes of exercise may result in different afferent feedback signals to the central nervous system and/or direct central effects via intrinsic brain modulations and conscious perceptions of effort ${ }^{3,4}$. The significant reduction of MEPs amplitudes when the subjects were submitted to high intensity protocol of exercise in our study may be related to intrinsic brain regulation mechanisms related to an increased internal sense of effort and/or mental stress and support the concept of central fatigue as a protective mechanism ${ }^{3}$. Alternatively, the lack of changes in excitability of the cortical cells after low/moderate exercises may be due to these modes of exercises have been unable to trigger the intrinsic brain regulation mechanisms.
The central response to the level of exercise intensity seems also depend on the levels of physical activity of subject. We demonstrated that behavior of the corticospinal excitability after dynamic exercise differs among the volunteers with different levels of physical activity according to IPAQ. Highly active individuals had significant depression in of excitability only after exercise of high-intensity. This finding demonstrates that in addition to the type, duration and intensity of exercise performed, the level of physical activity of the subjects may have influence the modulation of brain and spinal cord projections to exercising lower limb muscles during dynamic tasks like cycling. Cirilo, Lavender, Ridding, Semmler ${ }^{30}$ found that regular physical activity, primarily involving lower limb muscles, was accompanied by increased motor cortex plasticity in a small hand muscle compared with sedentary reinforcing that there are differences in behavior of brain and spinal cord according to level of physical activity.

In a previous study, it was showed that, the physically very active subjects had an increased neuroplastic response to a noninvasive brain stimulation protocol (Paired associate stimulation) when compared with sedentary individuals ${ }^{30}$. This result provides evidence that high levels of physical activity maintained over an extended period of time can enhanced the capacity for cortical plasticity. This supposed neuroplastic capacity enhancement associated to level of physical activity of individual may, in part, explain why physical activity has a positive effect on memory and executive function ${ }^{31}$. Another point to be considered is the production of lactate during exercise, studies have found that blood lactate concentration can influence the level of cortical excitability, mainly in the motor cortex ${ }^{32}$. It is suggested that during vigorous exercise, the accumulation of lactate in the blood reflects that the oxygen supply was inadequate to meet the energy requirements of the working muscle during the exercise ${ }^{33}$. Coco et al. ${ }^{32}$ showed that after acute and exhaustive exercise, the increases in blood lactate concentration are accompanied by decreased motor threshold, indicating an increase in the excitability of the motor cortex proportional concentrations of this metabolite. It is proven that individuals with better fitness level produce a smaller amount of blood lactate than irregular active subjects ${ }^{33}$, it is proven that individuals with better fitness level produce a smaller amount of blood lactate, this may be one explanation of the decrease in corticospinal excitability only in very active subject during highintensity exercise. However, these possibilities are speculative and more research is needed to confirm these findings.

The current study has some limitations that should be considered. Firstly, it is important to note that although the exercise was intended to be predominantly a lower limb exercise, the subjects were allowed to use their hands to grip the handle bars of the stationary ergometer. It is therefore theoretically possible, that minimal use of the hands for stabilisation during the intervention may have affected to the findings. Another limitation is that blood lactate levels may have influence in cortical excitability and different intensities of exercise may alter their concentration in the blood, thus analysis during and after different intensities of exercise may help to understand the responsiveness of corticospinal cells mainly in very active subjects. More studies, comparing the excitability corticoespinal 
in subjects with different levels of physical activity, and different representations of motor cortex (i.e., exercised muscle) are need to elucidate the mechanisms responsible for this effect.

In conclusion, our result highlights that changes in the corticospinal excitability depend on exercise intensity, duration and level of physical activity of subjects.

\section{References}

1. Amann M, Dempsey JA. Locomotor muscle fatigue modifies central motor drive in healthy humans and imposes a limitation to exercise performance. J. Physiol. 2008;586(1):161-73.

2. Brink-Elfegoun T, Kaijser L, Gustafsson T, Ekblom B. Maximal oxygen uptake is not limited by a central nervous system governor. J Appl Physiol. 2007;102(2):781-6.

3. Gandevia S. Spinal and supraspinal factors in human muscle fatigue. Physiol. Rev. 2001;81(4):1725-89.

4. Marcora S. Perception of effort during exercise is independent of afferent feedback from skeletal muscles, heart, and lungs. J Appl Physiol. 2009;106(6):2060-2.

5. Sidhu SK, Cresswell AG, Carroll TJ. Corticospinal responses to sustained locomotor exercises: moving beyond single-joint studies of central fatigue. Sports Medicine. 2013;43(6):437-49.

6. Périard JD, Caillaud C, Thompson MW. Central and peripheral fatigue during passive and exercise-induced hyperthermia. Med Sci Sports Exerc. 2011;43(9):1657-65.

7. Dempsey JA, Amann M, Romer LM, Miller JD. Respiratory system determinants of peripheral fatigue and endurance performance. Med Sci Sports Exerc. 2008;40(3):457-61.

8. Goodall S, Howatson G, Romer L, Ross E. Transcranial magnetic stimulation in sport science: A commentary. Eur J Sport Sci. 2014;14(sup1):S332-S40.

9. Kidgell DJ, Stokes MA, Castricum TJ, Pearce AJ. Neurophysiological responses after short-term strength training of the biceps brachii muscle. J Strength Cond Res. 2010;24(11):3123-32.

10. Sidhu SK, Cresswell AG, Carroll TJ. Motor cortex excitability does not increase during sustained cycling exercise to volitional exhaustion. J. Appl. Physiol. 2012;113(3):401-9.

11. Racinais S, Girard O, Micallef J-P, Perrey S. Failed excitability of spinal motoneurons induced by prolonged running exercise. Journal of neurophysiology. 2007;97(1):596-603.

12. Brasil-Neto JP CL, Hallett M. . Central fatigue as revealed by post-exercise decrement of motor evoked potentials. Muscle Nerve. 1994;17:713-19.

13. Taylor J, Gandevia S. Noninvasive stimulation of the human corticospinal tract. J. Appl. Physiol. 2004;96(4):1496-503.

14. Höllge J, Kunkel M, Ziemann U, Tergau F, Geese R, Reimers C. Central fatigue in sports and daily exercises. A magnetic stimulation study. Int J Sports Med. 1997;18(08):614-7.

15. Booth ML, Ainsworth BE, Pratt M, Ekelund U, Yngve A, Sallis JF, et al. International physical activity questionnaire: 12-country reliability and validity. Med Sci Sports Exerc. 2003;195(9131/03):3508-1381.

16. Matsudo S, Araújo T, Marsudo V, Andrade D, Andrade E, Braggion G. Questinário internacional de atividade fl sica (IPAQ): estudo de validade e reprodutibilidade no Brasil. Rev bras ativ fís saúde. 2001;6(2):05-18.

17. Borg GA. Psychophysical bases of perceived exertion. Med Sci Sports Exerc. 1982;14(5):377-81.

18. Hoffman BW, Oya T, Carroll TJ, Cresswell AG. Increases in corticospinal responsiveness during a sustained submaximal plantar flexion. J. Appl. Physiol. 2009;107(1):112-20.

19. McNeil CJ, Giesebrecht S, Gandevia SC, Taylor JL. Behaviour of the motoneurone pool in a fatiguing submaximal contraction. J. Physiol. 2011;589(14):3533-44.

20. Ross EZ, Middleton N, Shave R, George K, Nowicky A. Corticomotor excitability contributes to neuromuscular fatigue following marathon running in man. Exp. Physiol. 2007;92(2):417-26.

21. Verin E, Ross E, Demoule A, Hopkinson N, Nickol A, Fauroux $\mathrm{B}$, et al. Effects of exhaustive incremental treadmill exercise on diaphragm and quadriceps motor potentials evoked by transcranial magnetic stimulation. J Appl Physiol. 2004;96(1):253-9.

22. Samii A, Wassermann EM, Hallett M. Post-exercise depression of motor evoked potentials as a function of exercise duration. Electroencephalogr Clin Neurophysiol / Electromyogr Motor C. 1997;105(5):352-6.

23. Goodall S, González-Alonso J, Ali L, Ross EZ, Romer LM. Supraspinal fatigue after normoxic and hypoxic exercise in humans. J. Physiol. 2012;590(11):2767-82.

24. Sidhu SK, Bentley DJ, Carroll TJ. Locomotor exercise induces long-lasting impairments in the capacity of the human motor cortex to voluntarily activate knee extensor muscles. J Appl Physiol. 2009;106(2):556-65.

25. Takahashi K, Maruyama A, Hirakoba K, Maeda M, Etoh S, Kawahira K, et al. Fatiguing intermittent lower limb exercise influences corticospinal and corticocortical excitability in the nonexercised upper limb. Brain Stimul. 2011;4(2):90-6.

26. Rasmussen P, Nielsen J, Overgaard M, Krogh-Madsen R, Gjedde A, Secher $\mathrm{NH}$, et al. Reduced muscle activation during exercise related to brain oxygenation and metabolism in humans. J Physiol. 2010;588(11):1985-95.

27. McDonnell MN, Buckley JD, Opie GM, Ridding MC, Semmler JG. A single bout of aerobic exercise promotes motor cortical neuroplasticity. J Appl Physiol . 2013;114(9):1174-82.

28. Smith AE, Goldsworthy MR, Garside T, Wood FM, Ridding MC. The influence of a single bout of aerobic exercise on short-interval intracortical excitability. Exp. Brain Res. 2014;232(6):1875-82.

29. Samii A, Wassermann E, Ikoma K, Mercuri B, Hallett M. Characterization of postexercise facilitation and depression of motor evoked potentials to transcranial magnetic stimulation. Neurology. 1996;46(5):1376-.

30. Cirillo J, Lavender AP, Ridding MC, Semmler JG. Motor cortex plasticity induced by paired associative stimulation is enhanced in physically active individuals. J. Physiol. 2009;587(24):5831-42.

31. Cotman CW, Berchtold NC. Exercise: a behavioral intervention to enhance brain health and plasticity. Trends Neurosci. 2002;25(6):295-301.

32. Coco M, Alagona G, Rapisarda G, Costanzo E, Calogero RA, Perciavalle V, etal.Elevated blood lactate is associated with increased motor cortex excitability. Somatosens Mot Res. 2010;27(1):1-8. 
33. Myers J, Ashley E. Dangerous curves a perspective on exercise, lactate, and the anaerobic threshold. CHEST Journal. 1997;111(3):787-95.

\section{Corresponding author}

Katia Monte-Silva

Applied Neuroscience Laboratory, Universidade Federal de Pernambuco, Department of Physical Therapy. Av. Prof. Moraes Rego s/n, Recife, Brazil.

Email: monte.silvakk@gmail.com
Manuscript received on August 25, 2016

Manuscript accepted on November 15, 2016

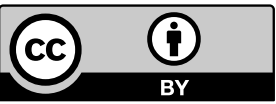

Motriz. The Journal of Physical Education. UNESP. Rio Claro, SP, Brazil - eISSN: 1980-6574 - under a license Creative Commons - Version 3.0 